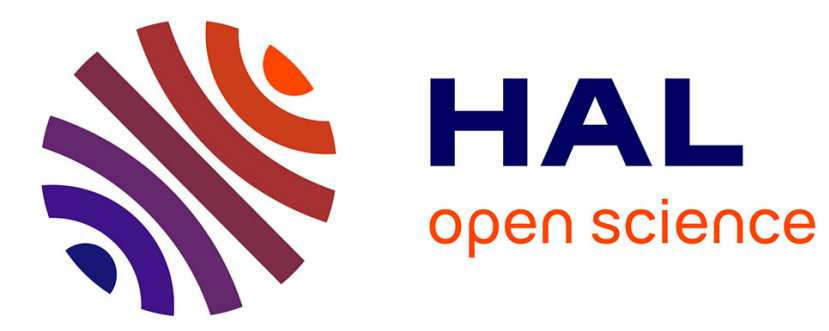

\title{
Précision et approximation dans l'évolution des conjonctions
}

Annie Bertin

\section{To cite this version:}

Annie Bertin. Précision et approximation dans l'évolution des conjonctions. L'information grammaticale, 2010, 125, pp.8-11. halshs-00732633

\section{HAL Id: halshs-00732633 \\ https://shs.hal.science/halshs-00732633}

Submitted on 15 Sep 2012

HAL is a multi-disciplinary open access archive for the deposit and dissemination of scientific research documents, whether they are published or not. The documents may come from teaching and research institutions in France or abroad, or from public or private research centers.
L'archive ouverte pluridisciplinaire HAL, est destinée au dépôt et à la diffusion de documents scientifiques de niveau recherche, publiés ou non, émanant des établissements d'enseignement et de recherche français ou étrangers, des laboratoires publics ou privés. 


\section{PRECISION ET APPROXIMATION \\ DANS L'EVOLUTION DES CONJONCTIONS}

Dans le langage naturel plus qu'ailleurs, « le vague est le vrai »

Robert Martin, Pour une logique du sens : 203 citant Renan, L'Avenir de la Science : 18

Précision et approximation seront envisagées ici non du point de vue du signifié mais du point de vue du signe, pris dans le changement linguistique. L'évolution diachronique d'une langue est en effet souvent décrite à travers la polarité précision-approximation : avancée vers une précision sémantique accrue du signe, par l'accroissement de l'isomorphisme dans les rapports signifiant-signifié, acquise par une baisse conjointe de la polysémie, de l'homonymie, de la synonymie, voire par une adaptation de la forme au sens. La perfection du signe, en tant qu'adéquation de la forme sinon au réel, du moins à la pensée, serait à chercher non à l'origine- l'histoire se lisant comme un écart grandissant du signe au référent et comme une dégénérescence-, mais comme la finalité de l'activité langagière à travers le temps. La catégorie de la conjonction constitue un objet sémantique paradoxal, fréquemment rapprochée des opérations logiques, ce qui invite à le décrire et à en décrire l'évolution selon les catégories, liées à la quantification mathématique, de la (simple) approximation et de la (grande) précision. C'est cette orientation diachronique, et la hiérarchisation implicite qu'elle exprime que l'on voudrait interroger et mettre en question. On reviendra d'abord sur l'opposition de l'approximation et de la précision en tant que participant d'une langue parfaite fantasmatique (1) ; on observera ensuite la dialectique de cette opposition dans l'évolution du système des conjonctions temporelles et causales (2), en centrant l'analyse sur l'évolution sémantique de puis que entre temporel et causal en Moyen Français (3). Si l'on peut s'interroger sur le caractère métaphorique ou métonymique du mécanisme qui fait passer une conjonction comme puis que, du temps à la cause, on sera amené à constater que le changement sémantique, qu'il soit lié aux associations d'idées suggérées par tel signe ou à une réanalyse de contextes ambigus, manifeste combien l'approximation, loin de constituer un obstacle à surmonter, est au cœur du fonctionnement du langage naturel. La précision quant à elle, si elle s'accroît à certains égards, dans la dynamique de la langue, joue moins comme terme téléologique du système, que comme conséquence de l'exigence de pertinence propre à l'acte de communiquer.

\section{LE FANTASME DE LA LANGUE PARFAITE}

Dans le langage ordinaire, approximation et précision sont pris dans des paradigmes axiologiquement opposés : d'un côté ce qui est vague, flou, ambigu, de l'autre ce qui précis, exact, net, juste, propre ; d'un côté le manque (de netteté, de clarté) qui dissimule et crée la possibilité d'induire en erreur, de l'autre ce qui est conforme au vrai, à l'essence, exempt de mélange. A travers ces oppositions lexicales se lit une ontologie occidentale que révulse la différance, au sens de Lévinas ou Derrida. On note que ces qualificatifs, qui s'appliquent au 
mot (juste, exact), au sens (propre), à l'expression (vague, floue), ne relèvent pas, à l'exception d'ambigu, originellement, du domaine linguistique : le flou est un terme pictural, l'approximation, au sens abstrait, vient du lexique scientifique. Tous ont en commun de sousentendre une conception spatiale du sens comme forme une et circonscrite, sorte de cible dont le langage, en tant qu'expression ne doit pas dévier, s'écarter.

L'écart est pourtant consubstantiel au langage naturel qui ne pourrait se ramener à une formalisation logique qu'à condition que le formalisme soit assez puissant pour intégrer ce caractère flou (Martin, 1997). La tropologie est ainsi fondée sur ce flou, ce vague du signe linguistique, dû aussi bien au caractère non discret du signifié («on glisse insensiblement d'une nuance de signification à une autre, d'une acception à une autre. (...) C'est précisément ce qui fait recourir Gustave Guillaume à l'hypothèse du cinétisme », Martin, 1983 : 27) qui rend possible un usage sélectif, métaphorique ou métonymique, qu'à la variabilité du contenu selon le locuteur. L'ambiguïté, l'impropriété, l'approximation ne sont que la rançon ou la face négative de la métaphore et de la métonymie, la classification tardive des tropes opérée par Donat, distribuant d'ailleurs les unes et les autres entre vitia virtutesque orationis (Desbordes 1988 : 86 sq). L'écart diachronique, en tant qu'il démultiplie la variation du sens pour le locuteur et pour le récepteur, rend sensible le caractère imparfait du langage naturel, si du moins l'on conçoit la perfection comme une coïncidence à un signifié fixe et univoque.

Quand Leibniz explore la possibilité d'une langue universelle jouant sur la logique mathématique et la structure syntaxique, les Idéologues constatent la variabilité du sens, liée au caractère discontinu et subjectif du langage, qui n'a pas les propriétés d'une langue algébrique :

\begin{abstract}
nous sommes réduits le plus souvent à des conjectures, à des inductions, à des approximations $[\ldots]$; nous n'avons presque jamais la certitude parfaite que cette idée que nous nous sommes faite sous ce signe par ces moyens, soit exactement et en tout la même que celle à laquelle attachent ce même signe, celui qui nous l'a appris et les autres hommes qui s'en servent. De là vient souvent que des mots prennent insensiblement des significations différentes, suivant les temps et les lieux, sans que personne se soit aperçu du changement : ainsi il est vrai de dire que tout signe est parfait pour celui qui l'invente, mais qu'il a toujours quelque chose de vague et d'incertain pour celui qui le reçoit [il y a plus] : je viens d'accorder que tout signe est parfait pour celui qui l'invente, mais cela n'est vrai que dans le moment où il l'invente, car quand il se sert de ce même signe dans un autre temps de sa vie, ou dans une autre disposition de son esprit, il n'est point du tout sûr que lui-même réunisse exactement sous ce signe la même collection d'idées que la première fois [...](Destutt de Tracy- Eléments d'idéologie II : 378-379 in Eco 1997 : 330. Nous soulignons.)
\end{abstract}

La transmission du signe linguistique (contrairement à celle du signe mathématique) induit inévitablement une variation qui, l'écartant de l'univoque, la condamne à l'imperfection :

(...) il est impossible que le même signe ait exactement la même valeur pour tous ceux qui l'emploient

[...]. Nous devons donc renoncer à la perfection (Destutt de Tracy- Eléments d'idéologie II : 378-379)

Et l'on peut conclure, avec U. Eco, que dès lors que l'on introduit le facteur temps dans le langage, il n'est pas possible de concevoir un langage qui soit fixe et un :

Si l'on décide que la pensée et le langage ont une genèse qui se dévide dans le temps (non seulement dans le temps reculé et préhistorique dont parle toute théorie de la naissance du langage, mais aussi le temps en 
devenir de notre histoire présente), toute tentative de penser à une langue philosophique est condamnée à l'échec. (Eco, $1997: 331)$.

L'approximation comme l'instabilité sont donc inhérentes au langage naturel en tant que praxis. Le terme présuppose un décalage entre deux entités, naissant d'un processus dynamique : le mot désigne, dans sa première attestation en français, le fait de rapprocher les deux bords d'une plaie, puis dans le langage scientifique, une «opération par laquelle on tend à se rapprocher de plus en plus de la valeur réelle d'une quantité ou d'une grandeur sans y parvenir rigoureusement». Transposée dans le domaine sémantique, l'approximation présupposera une béance du signe, imparfaitement résorbée. Plus que l'image (picturale) du flou pour rendre compte du \pm vrai dans le langage (Martin 1983), celle d'approximation présuppose une entité centrale vers laquelle vise un mouvement. Si l'approximation, tendant imparfaitement à la coïncidence, laisse une trace, elle n'en constitue pas moins un facteur d'économie cognitive.

La linguistique diachronique -ou dynamique- révèle l'irrémédiable hétérogénéité du sens. Le développement dans le temps peut cependant se lire de deux manières bien différentes, soit comme l'aliénation, par transmission successive d'un sens primitif, soit comme la conquête, à travers ce processus, d'une meilleure adéquation du système signifiant au sens. Loin de se lire comme la perte de la biunivocité parfaite d'un langage adamique, la dia-chronie est alors réduction de l'écart. Cette visée finaliste se lit particulièrement chez Guillaume et dans des travaux d'inspiration guillaumienne ${ }^{1}$.

L'approximation sémantique devient ainsi un moyen d'accroître la précision en système. La notion de macro-grammaticalisation développée, dans une toute autre perspective, par Christiane Marchello-Nizia, principalement pour les démonstratifs et les indéfinis (MarchelloNizia 2006, $231 s q$ ), rejoint des faits mis en lumière sur tel système de mots grammaticaux ; ainsi pour les conjonctions causales (Bertin 1997, la disparition des emplois complétifs voire relatifs de car (1-2), pourtant bien développés en Moyen Français, sans parler des emplois de quant en AF dans des constructions verbales que le FM rend par une complétive (3-4) ou de l'emploi massif en AF de que, alternant avec car, pour introduire une explication (5), participent d'une spécialisation accrue des outils d'intégration phrastique :

1) Et li roys Bauduins prioit car on l'assaille Le Bâtard de Bouillon 289

2) Et querroit le Bastart car il n'amoit noiant. ibid. 5045

3) Ja mais nul jour ne sera liés/ Et maudist tote s'aventure/ quant faite a tel engendreüre Sainte Marie l'Egyptienne T-94 (De co ke fist tel engendure- manuscrit C)

4) Pilates mout se merveilla / quant si petit don demanda. Boron R-457 (...s'en mervella moult de çou qu'il li ot si povre don demandé, Boron E-23-11)

5) Il est morz, que bien le veez. Boron R-535

\footnotetext{
${ }^{1}$ Cf. Guillaume 2004 : 17-18 cité par Verjans (2009:53) «Ce qui dans l'entier de la diachronie, reste de la diachronie non accomplie sert à l'accomplissement du système qu'on voit ainsi se renouveler historiquement et devenir, selon le destin propre de tout système, de plus en plus lui-même, c'est-à-dire système ". plus largement voir Verjans (2009 : chapitre 1) sur la visée finaliste de l'activité linguistique selon Guillaume en relation avec la conception energeienne du langage selon Coseriu.
} 
Parmi les «tendances évolutives «lourdes » » qui se dégagent de l'étude de la concession des origines au $\mathrm{XVI}^{\mathrm{e}}$ siècle Olivier Soutet met en lumière «sur le plan sémiologique (...) un mouvement de spécification s'exerçant (...) dans deux directions $»$ :

*D'abord la réduction des phénomènes de polysémie, soit par expulsion (ou presque) du champ de la concession de signes demeurant par trop polysémiques (pour), soit par spécialisation concessive d'autres signes (toutefois)

* ensuite, l'adéquation progressive de la morphologie au plan de la représentation: prédicativité systématique de quoi, non-prédicativité de que. (Soutet 1992, 234).

S'agissant de l'évolution du système des conjonctions, il est donc légitime de s'interroger selon les deux perspectives suivantes :

- La validité d'un schéma d'évolution des conjonctions allant vers la précision.

- Les divers facteurs d'approximation concourant au glissement sémantique des conjonctions : réinterprétation par le sujet parlant? réinterprétation sémantique et/ou syntaxique d'un énoncé par le récepteur, selon un schéma de misunderstanding?

C'est à ces deux points que l'on s'attachera successivement dans ce qui suit, en observant les emplois de puis que en Moyen Français, soit une conjonction qui manifeste le glissement bien connu du temporel au causal-justificatif, dans un état de langue bien documenté par les ressources électroniques largement disponibles ${ }^{2}$.

\section{LA DYNAMIQUE VERS LA PRECISION ET SES LIMITES}

La conjonction, lorsqu'elle n'est pas un simple outil d'intégration, marque une relation d'ordre temporel ou logique entre propositions. La précision sémantique peut résulter d'une modification de divers rapports : rapport du signifié au contexte, rapport entre les signifiés, rapport entre signifiant et signifié, rapport entre les signes. Dans le domaine de la causalité ou de la postériorité, on constate une tendance à une expression plus précise dans la mesure où le sens de la conjonction se dégage du contexte, où le signifié gagne en netteté, l'iconicité du système s'accroissant par élimination ou restriction de la polysémie et, ce qui y est lié, de la polycatégorialité. On devra toutefois se demander dans quelle mesure cette dynamique vers la précision concerne bien la langue et si elle n'est pas le reflet des genres textuels qui en sont les témoins. On doit aussi souligner que la polysémie ne disparait jamais, du moins pour certaines conjonctions, ce qui invite à s'interroger sur les facteurs qui favorisent ou au contraire bloquent la polysémie.

\subsection{La conjonction en diachronie : du syncatégorème au lexème ?}

Dans la grammaire médiévale, les termes invariables et grammaticaux comme les conjonctions et les prépositions ont en commun de «signifier avec » (consignificare), ils

\footnotetext{
${ }^{2}$ On s'appuie sur un corpus d'un peu plus de 1000 occurrences fournies par l'interrogation de la Base du Moyen français, qui fournit 1076 attestations de la suite puis que, dont il convient de retrancher une vingtaine d'énoncés comprenant la forme du verbe pouvoir ou l'adverbe puis redoublant le coordonnant et.
} 
n'ont pas de sens en eux-mêmes mais le tiennent de leur combinaison avec les autres termes de la phrase; quelles que soient les variations dans les classements grammaticaux et les théories du langage, d'Abélard à Guillaume d'Ockham s'opère la même distinction entre catégorèmes (le nom et le verbe, catégories morphologiquement variables et nécessaires au langage donc universelles) et les syncatégorèmes, qui participent selon Abélard, non de l'esse mais du bene esse du langage, moins nécessaire à la complétude de la phrase ; contrairement à la définition de la conjonction par Aristote comme un terme $\operatorname{asemos}^{3}$, on reconnait à la conjonction une signification, puisqu'elle contribue à produire le sens, à la différence d'une syllabe quelconque, mais cette signification ne peut être que «confuse et incertaine » (confusa per se et incerta) (Bertin, 1997: 8 citant Jolivet 1982, et Lusignan 1986). En donnant la priorité au sémantique sur le syntaxique dans la définition de la conjonction, la tradition grammaticale issue de l'Antiquité, limite son contenu à une fonction d'articulation et de révélateur de l'implicite, dans le prolongement de la définition de Denys le Thrace « La conjonction est un mot qui conjoint la pensée en ordonnant, et qui révèle l'implicite de l'expression » (éd. Lallot, 1989 : 65).

La relation causale ou temporelle entre propositions existe indépendamment du mot subordonnant qui conjoint celles-ci, si bien que la conjonction n'est pas indispensable, comme le rappelle Paul Imbs : «Le rapport temporel du verbe principal et du verbe subordonné est le support profond, permanent, du rapport de postériorité, dont nous rappellerons qu'in n'est qu'une variante du rapport d'achèvement; il est comme l'âme de la subordination de postériorité, au regard de laquelle la conjonction apparaît comme un simple accessoire, une sorte d'avertisseur supplémentaire pour lecteurs inattentifs ou férus de précision chronologique. »(Imbs, 1956: 335). On retracera donc volontiers, dans les grammaires historiques, l'histoire d'un système conjonctif à partir d'une première étape qui consiste en l'émergence d'une marque sémiologique de la notion : ainsi la postériorité, marquée en soi par les temps verbaux, est-elle soulignée, de manière redondante, par le mot quant, au sémantisme large. Viennent ensuite des signes spécifiques à la postériorité (puis que, de(s)puis que, des que, après (ce) que). En se dégageant du contexte, la conjonction tend à passer du statut de syncatégorème à celui de lexème tout en précisant son sémantisme.

\subsection{La conjonction en diachronie : d'un signifié flou à un signifié précis}

Si la précision sémantique est accrue par l'apparition, à partir du $12^{\mathrm{e}}$ siècle en français, de signes analytiques distinguant plus nettement, par elles-mêmes et non plus par le jeu des temps verbaux, la postériorité, celles-ci n'en expriment pas moins une valeur globale qui cumule souvent plusieurs valeurs ; plutôt que de présenter une polysémie, au sens où elles auraient des sens $\mathrm{a}^{1} \mathrm{a}^{2} \mathrm{a}^{3} \ldots \mathrm{a}^{\mathrm{n}}$ distincts, même s'ils appartiennent à un continuum, ces

\footnotetext{
${ }^{3}$ Aristote- Poétique, 20, 1456b 38-14657a 6 « La conjonction est un constituant de la parole dépourvu de sens qui est sans influence, ni positive, ni négative, sur la formation d'une unité signifiante de la parole résultant de la combinaison de plusieurs constituants, qu'on mette cette conjonction aux extrémités ou au milieu ; de plus, c'est un constituant qui ne peut se tenir seul en tête d'un énoncé(...). Ou encore, c'est un constituant de la parole dépourvu de sens, apte à créer une unité signifiante de la parole à partir d'au moins deux constituants qui soient eux-mêmes signifiants » (in Baratin, 1989 : 21)
} 
conjonctions semblent cumuler les différents effets de sens, dont l'un prédomine sans toutefois exclure les autres, l'analyse sémantique n'excluant pas la synthèse, selon l'étude de Paul Imbs : « dans la notion d'espace postérieur à un moment donné, l'ancien français place indistinctement à la fois les idées de départ («à compter de »), de commencement précoce (déjà «alors que » et d'un départ impliquant une limité finale de l'espace (« depuis que »); il laisse au contexte le soin de mettre en relief l'une ou l'autre de ces notions, sans que pour autant les deux autres notions complémentaires soient réellement absentes du plan de l'expression. » (Imbs, 1956 : 386). Aussi peut-on opposer, sous la permanence des formes, le système moderne de conjonctions exprimant la notion de postériorité, distinguant les notions avec netteté ${ }^{4}$ et précision $^{5}$, et celui de l'ancien français, l'histoire allant de la superposition sémantique à une signification circonscrite, « délimitée ${ }^{6}$.

\subsection{Iconicité, polysémie et polycatégorialité}

La superposition des significations est elle-même liée à la polysémie de la conjonction. Puis que, outre qu'il comporte en AF la double valeur causale et temporelle, offre dans le domaine temporel toute une palette, depuis son sens étymologique («après que ») jusqu'au sens très général de «quand», que l'on pourrait dire mutatis mutandis hyperonymique dans le domaine des relations temporelles; l'hésitation du traducteur moderne qui doit choisir entre après que, depuis que, une fois que, maintenant que, lorsque, quand serait à comparer à celui des traducteurs médiévaux du latin vers l'ancien français ; à propos de la traduction de cum + plus-que-parfait et cum + futur antérieur par puis que Imbs (1956 : 357) note ainsi : "s'agitil de la traduction renforcée d'une notion suggérée en latin par le seul rapport des temps verbaux, ou puis que n'est-il déjà plus qu'un synonyme, tout au plus expressif, de quant, en attendant qu'il ne s'emploie plus qu'avec une valeur causale ? » La valeur non spécifique de puis que au sens de «quand» peut déboucher comme pour le quant de l'AF sur une valeur d'opposition («alors que»), sur la valeur causale qui l'emportera ou sur la valeur hypothétique, et «peut se dégrader en morphème complétif (« de ce que ») (Imbs, 1956 : 404). Est alors mise en lumière les zones de recouvrement entre la catégorie du complément (essentiel) et de l'ajout ou complément circonstanciel. Si l'on maintient ces catégories, à la polysémie s'ajoute, du moins pour certaines conjonctions, la polycatégorialité. L'histoire s'écrit alors comme une marche vers l'iconicité : «Le parallélisme de quant et de puis que est alors complet, et l'on comprend que la langue ait fini par réagir contre un tel confusionnisme. L'on connaît la fin de l'aventure : quant retourne à sa pure valeur temporelle, puisque devient définitivement morphème de cause, depuis que opte pour sa valeur actuelle, et les morphèmes complétifs ne sont plus faits que d'éléments à sémantèse pauvre (que, de ce que, à ce que). »

\footnotetext{
${ }^{4}$ " ce système si rigoureux, et d'une netteté presque coupante, se dessine-t-il déjà en ancien français, ou celuici se meut-il décidément dans un univers pré-logique et anti-moderne ? » (Imbs, 1957 : 334 ).

${ }^{5}$ « La notion linguistique de la postériorité est assez fortement et surtout assez fermement structurée en français moderne pour qu'il soit possible de classer avec précision les expressions qui la traduisent. " (ibid.)

6 «plusieurs des expressions énumérées peuvent exprimer des notions multiples; c'est le cas en particulier, de puis que et des que - alors qu'en français moderne ces mêmes expressions ont une signification plus nettement délimitée» (ibid.).
} 
(ibid.). La syntaxe claire du FM émerge de ces emplois où la sélection du sens parmi le faisceau des sens virtuels repose sur le contexte ${ }^{7}$. Plus que dans d'autres systèmes linguistiques, il est alors difficile de prétendre accéder à la langue au-delà de ce que nous livre la parole qui la documente.

\subsection{Précision et « style »}

Or, les différents genres littéraires, donnant une part différente à la précision chronologique, amènent l'apparition de conjonctions différentes. La différence est surtout notable, selon l'étude de Imbs, entre le genre épique et la prose historique avec ses prolongements romanesques. Ainsi la conjonction après (ce) que connaît-elle un grand développement dans le genre historique : «son emploi est l'indice d'un certain souci de précision et d'exactitude, qui est étranger à une littérature visant avant tout au pittoresque et au pathétique » (Imbs, 1956: 371). La prose historique, et plus largement la prose narrative qui lui est liée génétiquement, accorde une place grandissante à la mise en perspective chronologique à l'aide de la subordination temporelle ${ }^{8}$ et d'une subordination temporelle toujours plus marquée du souci de précision". Si l'on admet que la normalisation du français s'est développée à partir de la norme écrite de la prose ${ }^{10}$, on perçoit l'importance de ce style pour l'étude du français en diachronie ; on devra cependant garder à l'esprit la question du plan où se situe le changement. Est-ce bien le système de la langue qui connait une avancée vers la précision rigoureuse de l'expression de la postériorité ou cette conséquence est-elle induite par

\footnotetext{
7 « Nous avons vu à quel point le Moyen Age se complaisait à ces notions synthétiques que seule la situation, c'est-à-dire la phrase, conduit à se déterminer dans le sens d'une des directions possibles. Mais ce choix exclut rarement toutes les autres possibilités, et une analyse sémantique conforme à l'esprit moderne est souvent une gageure. II n'ne reste pas moins que c'et de ces synthèses que se sont lentement dégagées les valeurs plus spécifiques de la syntaxe moderne ». (Imbs, 1957 : 407).

${ }^{8}$ " Quant à l'intellectualisation de la littérature, elle se traduit au plan de la syntaxe, par la multiplication des propositions subordonnées en général et des propositions temporelles en particulier, la subordination étant partout l'indice d'une pensée active, qui agence les faits disloqués, met de la perspective là où régnait la confusion des plans, réunit en discours ce qui n'était que tableau ». (ibid. 372)

${ }^{9}$ A propos du chroniqueur Joinville, Imbs (1957) écrit : « Ce qui frappe dans ce tableau, c'est la rigueur acquise par les relations temporelles. La valeur temporelle a définitivement, semble-t-il, pris le pas sur l'aspect. Les préoccupations logiques l'emportent visiblement sur le " style » : la grammaire s'intellectualise, et se constitue dans la mesure où elle devient un code. Des règles sont désormais possibles, le français devient l'instrument rigoureux qu'il tendra à être de façon de plus en plus systématique » (345).

${ }^{10}$ On rejoint sur ce point J. Kristeva : une tendance normative se fait jour qui, tout à la fois va contre toute polysémie et développe les outils conjonctifs marquant un rapport temporel et prédicatif. "Or, si on remontait dans l'histoire de la syntaxe française, on constaterait que la "normalisation de la phrase" française médiévale s'effectue dans la "prose", au travers d'une lutte contre la polysémie de la versification et des libertés syntaxiques de celle-ci. (...) Plus précisément encore, ces phrases prosaïques normatives, qui finiront par réglementer la grammaticalité du français, se constituent comme des "suites" dont les premiers mots sont des éléments conjonctifs liant les suites les unes aux autres de façon telle que le mouvement logique des enchaînements entre suites soit une "succession temporelle" qui recouvre plus ou moins, la relation logique entre "thème" et prédicat". " (Kristeva 1974 : 288 cité par Bertin, 1997 : 146).
} 
le changement des témoignages que l'on en a ? La marche de la langue vers la précision serait alors pour une bonne part illusion d'optique ${ }^{11}$, liée à la prédominance de la langue écrite dans les études linguistiques, dans celles d'histoire de la langue plus particulièrement. A cette limite à la précision s'ajoute le fait que la polysémie des conjonctions ne semble pas limitée exclusivement à la période ancienne de la langue.

\subsection{Pourquoi certaines conjonctions sont polysémiques, d'autres non ?}

La polysémie et la polycatégorialité sont certes en régression entre AF et FM, du moins dans la langue normée et l'on a pu établir par ailleurs un lien entre polysémie et ancienneté d'un subordonnant (Kortmann1997 et 1998). Toutefois on constate que certaines conjonctions restent polysémiques voire polycatégorielles, de plus celles qui sont apparues tardivement (à date littéraire, à la toute fin de l'AF et en MF) peuvent connaître un glissement sémantique (Bat-Zeev Shyldkrot 1989), analogue à celui de signes plus anciens, sans que cela touche toutes les conjonctions apparues à cette époque. On est donc amené à s'interroger sur les conditions de persistance de la polysémie et sur ce qui favorise ou bloque la polysémie. On constate, dans le domaine de la postériorité que, tout comme puis que, les conjonctions dès lors que, du moment que, maintenant que, une fois que peuvent se charger d'une valeur causale ; en revanche, après que reste exclusivement temporel. Si la postériorité est donnée comme un domaine source de l'expression de la cause justificative dans nombre de langues du monde (Heine et Kuteva, 2002) ${ }^{12}$, on voit que ce trait sémantique ne suffit pas. L'importante étude de Traugott et König (1991) dont l'argumentation s'appuie tout particulièrement sur l'évolution de sippan («from the time that») > since («puisque»), indique d'ailleurs que l'idée de séquentialité n'est pas en elle-même ce qui explique ce glissement, que l'on enregistre à travers diverses langues pour des mots au sémantisme temporel non séquentiel (latin dum, finnois kuna, allemand weil) et qui est bien plutôt lié à l'idée d'un recouvrement partiel entre procès ${ }^{13}$, que ceux-ci relèvent de l'évènement ou de l'état $^{14}$. Par leur sémantisme, certains marqueurs temporels sont donc aptes à exprimer la cause, d'autres non. Il convient toutefois de se demander si ce transfert s'explique par un changement déductif, d'ordre métaphorique, ou par un changement inductif, lié au contexte. C'est ce qu'on examinera pour finir.

\footnotetext{
${ }^{11}$ On peut élargir à tout le domaine des subordonnants et de la subordination la remarque de Rebecca Posner concernant le développement de l'hypotaxe: "It is often suggested that the history of French shows a change in complex sentence structure from parataxis to hypotaxis: this is clearly nonsensical if our starting point is Latin, which had sophisticated hypotactic construction. Perhaps the alleged change is illusory, depending on the type of Old Early evidence we have, rather than on the nature of the language." (Posner 1997, 348).

12 L'évolution de puisque à partir de posteaquam est mentionnée aux entrées since (275) et temporal> cause (291) avec le même commentaire : « This appears to be an instance of a widespread process whereby spatial and temporal markers are grammaticalized in specific contexts to markers of « logical » grammatical relations, such as adversative, causative, concern, concessive, and conditionnal relation ».

13 « It appears then that what is needed for a causal inference to arise is partial temporal overlap, not sequence » (Traugott \& König, 1991, 197).

${ }^{14}$ Ibid. 196-197.
} 


\section{POLYSEMIE ET EVOLUTION DE PUIS QUE EN MOYEN FRANÇAIS}

Le sens causal de puis que est très tôt réalisé (Bertin, 1997), au point qu'il peut faire oublier la très longue polysémie du terme. La spécialisation dans le sens causal, selon le tropisme vers la précision décrit ci-dessus, qui vaudrait pour le système de la langue lui-même, serait acquis selon Imbs (1956) au-delà de 1350. Pourtant l'examen d'un corpus assez vaste d'occurrences de Moyen Français montrent un sémantisme beaucoup moins homogène qu'attendu. La polysémie persistante de puis que permet alors d'étudier sur quoi jouent l'approximation (flou du message ou misunderstanding lié à l'inévitable polarité locuteur-récepteur ?) et la précision (évolution téléologique du système en diachronie ou pertinence communicative ?).

On observe la persistance, à côté d'un emploi nettement causal comme (6), où l'absence de successivité semble bloquer l'interprétation temporelle, des emplois nettement temporels («depuis que »), particulièrement avec des verbes d'aspect sémantiquement fini (naître, trespassser, (de)partir, venir) (7-8-9-10-11) :

6. je suis dampnez, Puis que la vierge m'est contraire, Qui aus autres est debonnaire. Miracle de l'esvesque que l'arcediacre murtrit, 1341, page 137

7. Onques mais, puis que je nasqui, En m' entente je ne vous vi, Et si ne sçay se c' estes vous. Miracle de l'enfant donné au diable, 1339, page 25

8. Puis que ton pére trespassa, Leesce en mon cuer ne passa Ne jour ne heure. Miracle de Saint Jehan Crisothomes, 1344, page 258.

9. Puis que vous partistes de nous Que vous advint? Miracle de la femme du roi du Portugal, 1342, page 167

10. Car puis que vous venistes ça Angoisse amére ne laissa Sa fille, ce sachiez pour voir, N'elle ne peut enfant avoir, Et jour et nuit adès travaille; Miracle de Saint Jehan Crisothomes, 1344, page 281.

Employé avec un verbe au subjonctif exprimant l'irréel, puis que équivaut à «pourvu que, sous réserve que » :

11. et aussi, puis que on les eust attenduz jusques au joindre, ilz eussent bien autant chassé que on eust sceü fouyr, et si sçavoient myeulx les chemins que nous Mémoires de Commynes, t.3, 1489, page 161.

La valeur proprement causale justificative est encore exclue dans des contextes exprimant une vérité générale, où puis que peut se rendre par si, quand, dès lors que non par puisque:

12. Car c' est celle qui ne scet tarder puis que on l' appelle. Miracle de l'evesque a qui Nostre dame s'apparut, 1348, page 59.

On observe enfin que la limite entre justification d'une assertion (« puisque »), constat d'une situation qui justifie l'assertion («maintenant que ») et complémentation du verbe (« de ce que ») n'est jamais totalement étanche ${ }^{15}$ :

\footnotetext{
${ }^{15}$ Cf. Harris 1986 inter alia.
} 
13. Ore, seigneurs, or loons Dieu, Puis que sommes en seur lieu; Miracle de Robert le Dyable, 1375, page 58

On ne peut donc pas dire que puisque ait atteint en MF une valeur unique «précise ». De plus un très grand nombre d'occurrences offre la possibilité d'une double lecture, temporelle et/ou causale; dans une phrase hors contexte, on hésitera, si l'on veut traduire en FM, entre après que et puisque (14), une fois que et puisque (15) :

14. Papirius mena en la bataille ces chevaliers, puis que il les ot en telle maniere resné et apparellié. Pierre Bersuire, les Décades de Titus Livius I, 9, 1354, page 73.

15. Et, puis que chien est enragié de l'une des neuf rages, onques nul ne peut garir ne jamés n'en garira. Gaston Phébus, Livre de chasse, 1387, page 114.

C'est seulement la visée que l'on donne au message qui fera choisir l'une ou l'autre interprétation : que ce soit dans un récit (14) ou dans un exposé didactique (15), s'il s'agit seulement d'exposer la succession des procès, de manière objective, on optera pour un lien temporel (après que, une fois que), au contraire si l'on estime que la narration a aussi une fonction de commentaire par le narrateur de son récit («c'est bien normal que ce soit lui qui les emmène au combat puisque c'est lui qui les y a préparés par ses exhortations ») ou que la proposition constitue, non seulement l'exposé d'un fait réalisé dans le temps («quand»), mais l'antécédent d'un enchaînement inéluctable ("une fois que, dès lors que ») voire l'antécédent de cet enchaînement selon une causalité que l'on présente comme admise de l'interlocuteur («puisque »), on optera pour puisque. On peut faire cette double analyse pour de nombreux passages, selon le degré de subjectivité et de force argumentative que l'on juge pertinent :

16. Ore faut il, puis que le vallet nouvel scet aler en queste et destourner cerf et sangler, qu'il le saiche bien et a point laisser courre. Gaston Phébus, le Livre de chasse, 1387, page 172.

17. Et lors ledit Jehan respondi que il avoit bien autrefois ouy dire que puis que on c' estoit confessez d' un larrecin, que l' en n' en povoit plus riens savoir; Registre criminel du Châtelet, t.2, 1389, page 283.

Le discours présente également de nombreux cas où l'on peut rendre puis que aussi bien par une conjonction temporelle qui établit un constat («maintenant que ») que par le puisque moderne :

18. Seigneurs, sachiez j' ay grant talent, Puis que j' ay dite ma complie, D' aler couchier, que je n' oblie A relever a mie nuit. Miracle de l' evesque que l'arcediacre murtrit, 1341, page 111.

19. Or puis je bien penser de vray Que soussier ne me fault mie, Puis que l' evesque a perdu vie, Que je n' aie briefment la croce, Et seray de la haulte boce A ceste foiz. Ibid. page 116.

20. Bien aviser nous en convient, Puis que nous en sommes chargié. Ibid. page 125.

21. Puis que vous voy saine et vivant, Il ne me chaut du remanant, Car j'ay assez. Miracle de la femme du roy de portigal, 1342, page 186.

22. Puis que Dieu vous a espiré A ce faire et voloir donné De sainte eglise exaucier, A mon pouoir vous vueil aidier. Miracle de la femme du roy de portigal, 1342, page 202 
23. Par amour, or nous en alons, Puis que noz offrandes sont faites: On verra maishui moult de sectes De gens venir. Miracle de la natavite nostre seigneur Jhesu Crist, 1343, page 230.

La double possibilité est d'ailleurs encore possible en FM, et c'est seulement selon que l'on juge admissible ou non d'un point de vue pragmatique de dire ce qui est connu que se fera le choix, la justification par puisque permettant de faire de la proposition non une redite ou un redoublement d'une information présente dans la situation d'énonciation, mais un outil argumentatif.

Le glissement du temporel au causal tient donc d'un choix pour rendre le message pertinent et accroître son pouvoir d'information. Il est lié non seulement à des implications sémantiques générales, jouant du recouvrement des notions de temps et de cause, mais au principe général selon lequel on parle pour faire le plus possible, avec pertinence ${ }^{16}$. Plus qu'à la discontinuité de la communication, qui entraînerait une réanalyse sémantique ou syntaxique, par le récepteur $^{17}$, le changement sémantique reposerait principalement sur un renforcement du pouvoir informationnel (strengthening of informativeness) de son message par le locuteur (Traugott \& König, 1991 : 210). Cet accroissement de la valeur informationnelle du message, qui passe, dans le changement diachronique, du plan contextuel au plan sémantique, participe certes d'une exigence de précision du langage. On voit toutefois que ce souci de précision procède du fonctionnement général de la communication linguistique, non d'une finalité inhérente au système linguistique lui-même.

\section{CONCLUSION}

Les relations de la diachronie à la précision et à l'approximation sont finalement paradoxales. L'approximation, en tant qu'elle rend possible les mécanismes métonymiques et métaphoriques, est la condition même du changement; l'évolution diachronique ne peut l'éliminer. Dans la mesure où l'évolution, du moins dans le domaine des conjonctions,

\footnotetext{
${ }^{16}$ L'étude de corpus nous fait rejoindre les conclusions de Traugott \& König $(1991: 191)$ : «The fundamental process we see at work is a principle of informativeness or relevance, essentially the principle: Be as informative as possible, given the need of situation". Sur le rôle primordial de la pragmaticalisation dans le changement diachronique, voir aussi Simone (2007).

${ }^{17}$ Cf. Hoenigswald, 1992 : 96: “ If, according to Stöcklein and Sperber [so, roughly, writes Leumann], the change of a word meaning rests on a given sentence context, it is certainly legitimate to start with those words whose peculiar syntactic function links uniquely to sentence-structure -that is with conjunctions and sentence-connecting particles. Take the wellknown French car 'for, because', from Latin quare 'why': here the radical semantic reversal can only be due to the discontinuity between speaker and hearer. However widespread the use of quare in rhetorical self-interruptions may have been, no speaker who still feels that etymological meaning 'why', can possibly interpret it as 'because'; only the hearer who no longer "kows" the etymological meaning will do so when he seecks to understand an uttered sentence like heri non veniquare- aegrotus eram 'yesterday I did not come -why- I was sick" (Manu Leumann, "Zum Mechanismus des Bedeutungswandels”, Indogermanische Forschungen 1927, 14:105-18 repris dans Kleine Schriften, Zürich, Stuttgart, 1959, 286-95.)
} 
procède du besoin de donner au message sa valeur informative maximale, à travers un processus d'ordre métonymique, l'approximation est alors le vecteur de la précision.

Annie BERTIN

Université Paris Ouest Nanterre La Défense- UMR Modyco

\section{BIBLIOGRAPHIE}

BARATIN M. (1989), La Naissance de la syntaxe à Rome, Minuit.

Base de Moyen Français : www.atilf.fr

BAT-ZEEV SHYLDKROT H. (1989), "Conjonctions et expression temporelle-causale en français". Folia Linguistica Historica, X: 1-2, 263-281.

BAT-ZEEV SHYLDKROT, H. (1993), "Sur le rapport temporel-causal dans les subordonnées. Le cas de en attendant que - attendu que ". Travaux de Linguistique, XXVII, 113-123.

BERTIN A. (1997), L’Expression de la cause en ancien français, Genève, Droz.

BERTIN A., (2003), “ Les connecteurs de cause dans l'histoire du français - contradictions du changement linguistique ", Verbum, XXV, 3, 263-276.

DESBORDES F. (1988), «Homonymie et synonymie d'après les textes théoriques latins », in L'ambiguité, cinq études historiques réunies par Irène Rosier, Presses universitaires de Lille, 51-102.

ECO U. (1997) [1994¹], La recherche de la langue parfaite, Paris, Editions du Seuil.

GUILlAUME G. (2004), Essais et mémoires de Gustave Guillaume. Prolégomènes à la linguistique structurale II. Discussion et continuation psychomécanique de la théorie saussurienne de la diachronie et de la synchronie, publiés sous la direction de R. Lowe, Québec, Les Presses de l'Université Laval.

HARRIS M. (1986), “Aspects of subordination in English and other languages ”, Bulletin of the John Rylands University Library of Manchester, 69 :1, 195-209.

HEINE B. \& KUTEVA T. (2002), World lexicon of grammaticalization, Cambridge, Cambridge University Press.

HOENIGSWALD H. M. (1992) "Semantic change and 'regularity' : A legacy of the past", in Kellermann, Günter et Michael Morrissey, éd. 1992. Diachrony within synchrony : language history and cognition. Papers from the international symposium at the University of Duisburg, 26-28 Mars 1990, Frankfurt-am-Main, Peter Lang, 85-105. 
KORTMANN B. (1997), Adverbial Subordination. A Typology and History of Adverbial

Subordinators Based on European Languages, Berlin- New York, Mouton de Gruyter.

KORTMANN B., 1997a, "The evolution of the adverbial subordinators in Europe », in Schmid M. S., Austin J. R., Stein D. (eds.), Historical Linguistics, Amsterdam/Philadelphia, Benjamins, pp. 213-228.

IMBS P. (1956), Les propositions temporelles en ancien français, Strasbourg, Publications de la Faculté des Lettres de Strasbourg.

JOLIVET J. (1982), Arts du langage et théologie chez Abélard, $2^{\text {de }}$ éd. augmentée, Paris, Vrin.

KRISTEVA J. (1974), La Révolution du langage poétique, Paris, Le Seuil.

LUSIGNAN S. (1986), Parler vulgairement, Paris, Vrin- Les Presses de l'Université de Montréal.

MARTIN R. (1983), Pour une logique du sens, Paris, PUF.

POSNER R. (1997), Linguistic change in French, Oxford, Clarendon Press.

SIMONE R. (2007), "Constructions : types, niveaux, force pragmatique », A la quête du sens- Etudes littéraires, historiques et linguistiques en hommage à Christiane MarchelloNizia, textes réunis par Céline Guillot, Serge Heiden, Sophie Prévost, Lyon, ENS Editions, 137-159.

TRAUGOTT E. C., KÖNIG E. (1991), «The semantics-pragmatics of grammaticalization revisited », in Traugott E.C, Heine B. éd., 1991, Approaches to grammaticalization, vol.1, Amsterdam-Philiadelphia, Johns Benjamins, 189-218.

VERJANS T. (2009), Essai de systématique diachronique: Genèse des conjonctions dans l'histoire du français (IX ${ }^{\mathrm{e}}-\mathrm{XVII}^{\mathrm{e}}$ siècles), thèse de l'Université Paris IV-Sorbonne. 